\title{
SEGMENTATION OF VISCERAL ADIPOSE Tissue Causing Central Obesity using DEEP LEARNING ON ABDOMINAL MRI
}

\author{
B Sudha Devi ${ }^{1}$ and Dr.D.S Misbha ${ }^{2}$ \\ ${ }^{1}$ Department of Computer Science, Nesamony Memorial Christian College, \\ Marthandam, India \\ ${ }^{2}$ AP, Department of Computer Science, Nesamony Memorial Christian \\ College, Marthandam, India
}

\begin{abstract}
In recent years, obesity is highly prevalent and is related with increased risk of many diseases. The distribution of abdominal adipose tissue plays a major role to assess central obesity. The basic objective of this study is to develop a novel method for automatic segmentation of visceral adipose tissue(VAT) and subcutaneous adipose tissue(SAT) from abdominal Magnetic resonance imaging(MRI) slices which is implemented in two steps. First, clustering of image is done to classify MR image into adipose tissue and non-adipose tissue. Second, after clustering the image, segmentation is done to separate VAT and SAT by a convolutional deep neural network. Sixty five MR images have been used in this study where deep learning techniquet have been adopted for the segmentation of VAT and SAT. The proposed and the manual measurements produced the Dice scores of 0.97 and 0.96 for SAT and VAT respectively. The experimental results show that the deep learning method produces better segmentation results with high accuracy.
\end{abstract}

\section{KEYWORDS}

Obesity, Visceral adipose tissue (VAT), subcutaneous adipose tissue (SAT), Magnetic resonance imaging (MRI), deep learning

\section{INTRODUCTION}

Visceral adipose tissue and subcutaneous adipose tissue are the two types of abdominal adipose tissues seen in humans. Subcutaneous adipose tissue is the fat that is stored beneath the skin where as visceral adipose tissue is the fat that is deposited between the internal organs in the abdominal cavity. Too much of any body fat is bad for our health. But compared to subcutaneous fat, visceral fat is more likely to raise the risk for serious medical issues like Alzheimer's disease, type 2 diabetes, cardiovascular diseases, musculoskeletal disorders, some cancers and high cholesterol. The excess amount of VAT in the human body is known as obesity. Although Body mass index (BMI), waist circumference and waist-hip ratio (WHR) has been used as a traditional measure of obesity, the results are approximate in obese and underweight individuals. Also, these measurements do not differentiate SAT and VAT [1]. At present, obesity can be determined by the amount of VAT in the abdominal region [2]. Apart from the traditional measures such as BMI and waist-hip ratio, other methods like bioelectrical impedance, dual energy X-ray absorptiometry, CT and MRI based techniques were used to measure abdominal fat. Recently, imaging modalities like CT and MRI are widely used to assess abdominal adipose tissue. The quantification of VAT 
from CT and MRI is considered to be more accurate and consistent measure of the distribution of body fat. On the other hand, CT is exposed to high radiation and so rarely used. MRI can measure SAT and VAT without any side effects. As a result, several unsupervised methods for the segmentation of adipose tissue are being developed. There are several studies that provides evidences on the accuracy of automated segmentation and quantification using deep learning. Different models like fully convolutional network (FCN), U-net neural network model, augmented active shape model and multi-atlas segmentation model uses different algorithms for the segmentation process[3].

\section{RELATED WORKS}

CT and MRI images are commonly used to segment adipose tissue. Deep learning using convolutional neural networks have recently gained importance in the literature for solving problems in a variety of fields, including image recognition, classification, and segmentation. Deep learning algorithms depend on large cohorts of training data to extract important features from the targets for new predictions.

Hui et al. [4] proposed an MRI based approach for segmenting VAT and SAT using spoke template and intensity based filtering. Sadananthan et al.[5] proposed a graph theoretic segmentation algorithm to segment VAT and SAT from MR images. A threelevel convolutional neural network was suggested by Wang et al.[6] for the segmentation of SAT and VAT.

Deep learning concept was used by Weston et al. [7] to perform abdominal VAT and SAT segmentation. Langner et al. [8] proposed a three channel U-Net architecture for the segmentation of SAT and VAT using abdominal MR images of humans. Ning Shen et al. [9] presented a quantification of subcutaneous and visceral adipose tissue from MRI based on machine learning. Young Jae Kim [10] proposed a method using separation mask to detect SAT and VAT quantities from CT images. For the segmentation of VAT and SAT on CT images, Grainger et al.[11] used the deep learning concept based on the U-Net architecture. Most of the MRI based adipose tissue segmentation algorithms concentrates on raw features like intensity, shape and location other than the intrinsic features of tissues. Fuzzy c-means algorithms [12] and K-means clustering algorithms [13] groups the pixels by considering the intensity features. Currently, multi-echo Dixon technique is also used for the assessment of adipose tissue. In this technique, to derive chemical shift-separated water and fat images from multiple source images acquired at different echo times a spectral model is used. To segment VAT and SAT in the abdominal region using DIXON MRI images, Estrada et al.[14] proposed a deep learning pipeline .

In this study, we introduce a novel method for VAT segmentation using fuzzy c-means algorithm and convolutional deep neural network that results in high performance and accuracy.

\section{PROPOSED METHODOLOGY}

Convolutional neural network is a class of deep neural network applied to analyze visual imagery in medical applications. As the deep learning method is independent of hand 
crafted features, it is able to learn the features from the image data that cannot be extracted by the image analyst. In this proposed work, segmentation of VAT and SAT was done by the U-Net architecture which is a convolutional deep neural network. The overall representation of the proposed $\mathrm{CNN}$ is shown in figure 1.

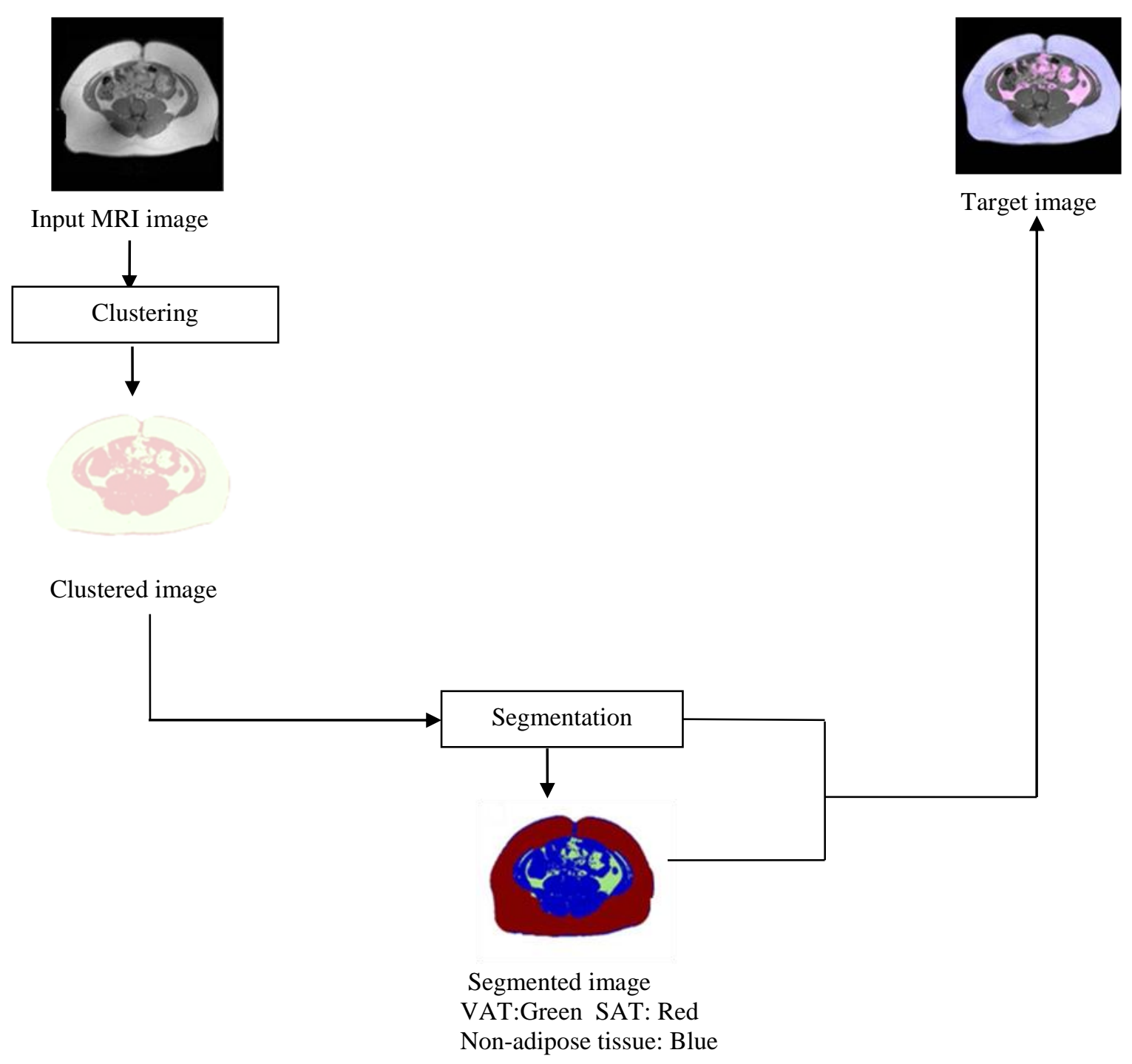

Figure 1. Flow diagram of the proposed $\mathrm{CNN}$ based segmentation method

In the first step, clustering is done to classify the MR image into adipose and non-adipose tissues. This is done using fuzzy c-means (FCM) algorithm as it is highly efficient and does not require any previous information. During the iterations of FCM, the energy function that is to be minimized is defined as

$$
P(x, c)=\sum_{i=1}^{K} \sum_{n=1}^{N}\left|x_{i}-c_{n}\right|^{2}
$$


where $\mathrm{K}$ represents the total number of voxels of the MR image, $\mathrm{N}$ is the number of clusters $($ Here $\mathrm{N}=2), \mathrm{x}_{\mathrm{i}}$ is the voxel intensity and $\mathrm{c}_{\mathrm{n}}$ is the center intensity of the $\mathrm{n}^{\text {th }}$ cluster. For the above function, iterative optimization was done with the updation of fuzzy cluster membership of each voxel and the center of each cluster. When the centers and the membership of the cluster were stabilized, or the allowed iteration was reached, the continuing iteration will be stopped. Figure 2 displays the result of clustering of an MR image. The grey color indicates adipose tissue and the black color indicates non-adipose tissue.

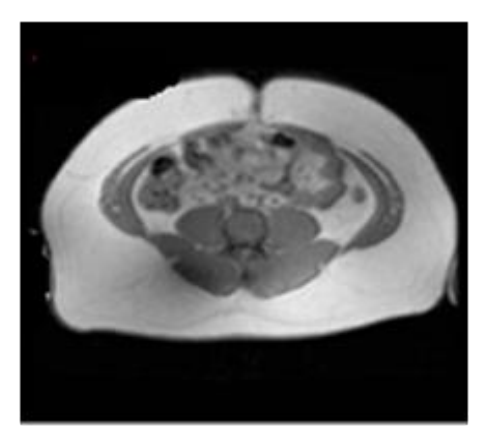

a

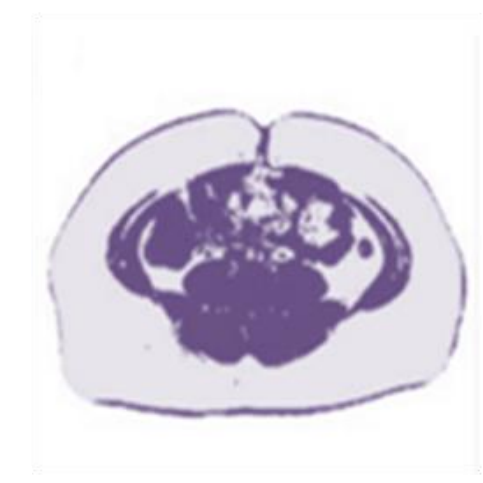

b

Figure 2. a) Original MRI slice b) Classification of adipose and non-adipose tissue using FCM clustering

In the second step, U-Net, a convolutional deep neural network was employed for the segmentation of VAT. The architectural design of U-Net consists of several cascading layers of learnable convolutional filters . The organization of the system includes five downsampling and five up-sampling layers, that minimized the input image resolution of $512 \times 512$ to a $16 \times 16 \times$ 196 representation which is upsampled into a $512 \times 512 \times 6$ output. A hyperbolic-tangent activation function(tanh), that normalized the values to the range of -1 to +1 , follows each step with two successive $3 \times 3$ convolutions. This is followed by max-pooling whose kernel size is $2 \times$ 2 pixels. In the next half of the network, a $2 \times 2$ nearest-neighbor interpolation performs the upsampling operations which is followed by two convolutional layers. The output of the convolutional kernel from the encoding step of the network is concatenated with each corresponding decoding step, which helps to preserve the detail of the original image. The final layer consisted of a convolution with a $1 \times 1 \times 2$ kernel which is followed by a sigmoid function, that provides an output score for both tissue types. The final segmentation is accomplished through the selection of tissue type with the highest score for each pixel. The U-Net deep learning model is combined with fuzzy c-means algorithm to classify adipose tissue and segment VAT. The segmentation results after clustering an MRI slice is shown in figure 3. 


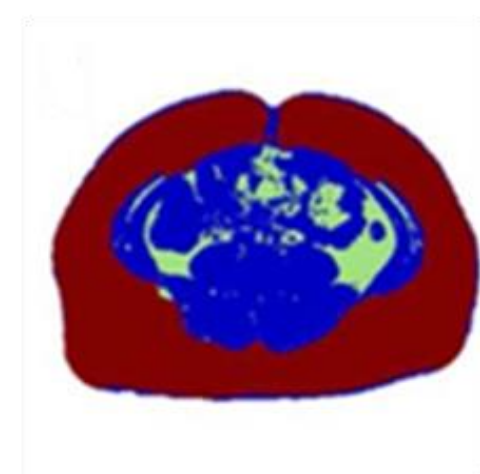

$\mathbf{a}$

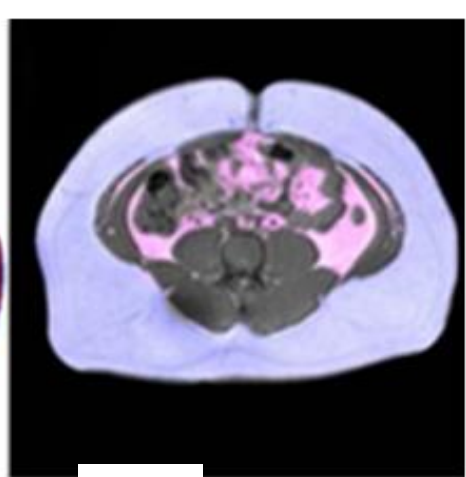

b

Figure 3. a) Segmentation result in which green coloured area indicates VAT, red coloured area indicates SAT and blue coloured area indicates non-adipose tissue b) Final segmented output from original MRI slice-pink coloured area represents VAT and blue coloured area represents SAT .

\section{EXPERIMENTAL RESULTS}

The cohort for this study includes 65 non-contrast MRI datasets collected for standard CAC scoring. These datasets were collected randomly from the prospective EISNER trial obtained at NIMS Medical Center. The patient population included asymptomatic subjects without any previous history of coronary artery disease but with cardiovascular risk factors. The population is shown in Table 1. There were no scans left because of low image quality in image or artefacts.. The total number of transverse slices per scan was 55 , and each axial slice had a dimension of $512 \times 512$ pixels of $0.684 \mathrm{~mm} \times 0.684 \mathrm{~mm}$. To implement this proposed model, a workstation with Dual Intel Xeon Processor E5-2630 v2 (Six-Core HT, 2.6GHz Turbo, $15 \mathrm{MB}$ ), 4 GB NVIDIA Quadro K5000, 1x8GB DDR3, 1TB 7200RPM SATA, and Windows 10 Pro workstation is used as a platform. Python 3.7 and open source machine learning libraries such as TensorFlow 1.13.1, Keras 2.2.4, and Scikit-learn 0.20.3 have been used to develop its working model.

Table 1. Subject Characteristics

$\begin{array}{ll}\text { Subjects } & 65 \\ \text { Male } & 40 \\ \text { Female } & 25 \\ \text { Age (years) } & 35 \text { to } 65 \\ \text { Diabetes } & 10 \\ \text { Hypercholesterolemia } & 40\end{array}$

The segmentation results produced by the proposed model were compared against the results generated by manual segmentation method. The performance was validated using Dice score and Jaccard score metrics. Let $\mathrm{L}$ and $\mathrm{M}$ indicate the manual segmentations and the CNN based segmentations respectively, then, Dice score is defined as, 
Jaccard score is defined as,

$$
\mathrm{DC}=\frac{2\|\operatorname{LnM}\|}{\|L\|+\|M\|}
$$

$$
\mathrm{JC}=\frac{\|L \cap M\|}{\|L U M\|}
$$

The convolutional deep neural network based segmentation method shows good correlation with manual segmentation. Table 2 shows the results of the proposed CNN and manual method. It can be seen from the table that the proposed method produced high Dice score value(VAT-0.96 and SAT- 0.97). The Jaccard scores of VAT and SAT are 0.95 and 0.96 respectively which is also high compared to manual segmentation. Figure displays the graphical representations of the performance evaluation results.

Table 2. Performance evaluation of manual segmentation and proposed CNN

\begin{tabular}{|l|c|c|c|c|}
\hline \multicolumn{1}{|c|}{$\begin{array}{c}\text { Evaluation } \\
\text { method }\end{array}$} & $\begin{array}{c}\text { VAT } \\
\text { Dice score }\end{array}$ & $\begin{array}{c}\text { SAT } \\
\text { Dice score }\end{array}$ & $\begin{array}{c}\text { VAT } \\
\text { Jaccard score }\end{array}$ & $\begin{array}{c}\text { SAT } \\
\text { Jaccard } \\
\text { score }\end{array}$ \\
\hline $\begin{array}{l}\text { Manual } \\
\text { method }\end{array}$ & 0.95 & 0.95 & 0.93 & 0.95 \\
\hline Proposed CNN & 0.96 & 0.97 & 0.95 & 0.96 \\
\hline
\end{tabular}

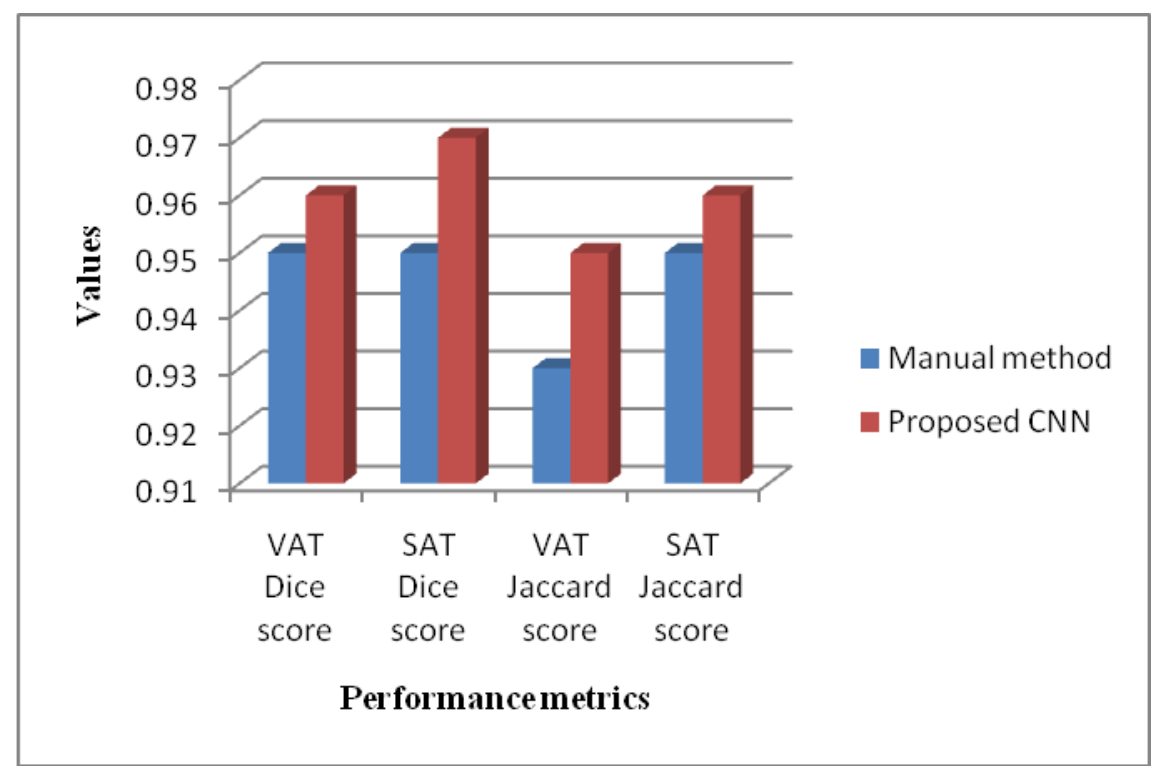

Figure. 5. Segmentation performance of manual segmentation and proposed CNN 


\section{CONCLuSiON}

The proposed deep learning method segments the visceral adipose tissue and subcutaneous adipose tissue from MR images of the abdomen with high accuracy. The performance is also high compared to manual segmentation. By segregating VAT and SAT, this work helps the clinicians to access central obesity and the risks associated with it. This can be further extended to handle the datasets with less training time.

\section{REFERENCES}

[1] D. Wald, B. Teucher, J. Dinkel, R. Kaaks, S. Delorme, H. Boeing, K. Seidensaal, H. Meinzer, T. Heimann, Automatic quantification of subcutaneous and visceral adipose tissue from whole-body magnetic resonance images suitable for large cohort studies, J. Magn. Reson. Imaging. 36 (2012) 1421-1434. https://doi.org/10.1002/jmri.23775.

[2] S. Hussein, U. Bagci, A. Green, A. Watane, D. Reiter, X. Chen, G.Z. Papadakis, B. Wood, A. Cypess, M. Osman, Automatic Segmentation and Quantification of White and Brown Adipose Tissues from PET/CT Scans, IEEE Trans. Med. Imaging. $36 \quad$ (2017) 734-744. https://doi.org/10.1109/TMI.2016.2636188.

[3] H.J. Park, Y. Shin, J. Park, H. Kim, I.S. Lee, D.-W. Seo, J. Huh, T.Y. Lee, T. Park, J. Lee, K.W. Kim, Development and Validation of a Deep Learning System for Segmentation of Abdominal Muscle and Fat on Computed Tomography, Korean J Radiol. 21 (2020) 88. https://doi.org/10.3348/kjr.2019.0470.

[4] S.C.N. Hui, T. Zhang, L. Shi, D. Wang, C.-B. Ip, W.C.W. Chu, Automated segmentation of abdominal subcutaneous adipose tissue and visceral adipose tissue in obese adolescent in MRI, Magnetic Resonance Imaging. 45 (2018) 97-104. https://doi.org/10.1016/j.mri.2017.09.016.

[5] S.A. Sadananthan, B. Prakash, M.K.-S. Leow, C.M. Khoo, H. Chou, K. Venkataraman, E.Y.H. Khoo, Y.S. Lee, P.D. Gluckman, E.S. Tai, S.S. Velan, Automated segmentation of visceral and subcutaneous (deep and superficial) adipose tissues in normal and overweight men: Automated Segmentation of Adipose Tissue, J. Magn. Reson. Imaging. 41 (2015) 924-934. https://doi.org/10.1002/jmri.24655.

[6] Z. Wang, Y. Meng, F. Weng, Y. Chen, F. Lu, X. Liu, M. Hou, J. Zhang, An Effective CNN Method for Fully Automated Segmenting Subcutaneous and Visceral Adipose Tissue on CT Scans, Ann Biomed Eng. 48 (2020) 312-328. https://doi.org/10.1007/s10439-019-02349-3.

[7] A.D. Weston, P. Korfiatis, T.L. Kline, K.A. Philbrick, P. Kostandy, T. Sakinis, M. Sugimoto, N. Takahashi, B.J. Erickson, Automated Abdominal Segmentation of CT Scans for Body Composition Analysis Using Deep Learning, Radiology. $290 \quad$ (2019) 669-679. https://doi.org/10.1148/radiol.2018181432.

[8] T. Langner, A. Hedström, K. Mörwald, D. Weghuber, A. Forslund, P. Bergsten, H. Ahlström, J. Kullberg, Fully convolutional networks for automated segmentation of abdominal adipose tissue depots in multicenter water-fat MRI, Magn. Reson. Med. 81 (2019) 2736-2745. https://doi.org/10.1002/mrm.27550.

[9] N. Shen, X. Li, S. Zheng, L. Zhang, Y. Fu, X. Liu, M. Li, J. Li, S. Guo, H. Zhang, Automated and accurate quantification of subcutaneous and visceral adipose tissue from magnetic resonance imaging based on machine learning, Magnetic Resonance Imaging. 64 (2019) 28-36. https://doi.org/10.1016/j.mri.2019.04.007.

[10] Y.J. Kim, J.W. Park, J.W. Kim, C.-S. Park, J.P.S. Gonzalez, S.H. Lee, K.G. Kim, J.H. Oh, Computerized Automated Quantification of Subcutaneous and Visceral Adipose Tissue From Computed Tomography Scans: Development and Validation Study, JMIR Med Inform. 4 (2016) e2. https://doi.org/10.2196/medinform.4923. 
[11] A.T. Grainger, A. Krishnaraj, M.H. Quinones, N.J. Tustison, S. Epstein, D. Fuller, A. Jha, K.L. Allman, W. Shi, Deep Learning-based Quantification of Abdominal Subcutaneous and Visceral Fat Volume on CT Images, Academic Radiology. (2020) S1076633220304268. https://doi.org/10.1016/j.acra.2020.07.010.

[12] A. Zhou, H. Murillo, Q. Peng, Novel segmentation method for abdominal fat quantification by MRI, J. Magn. Reson. Imaging. 34 (2011) 852-860. https://doi.org/10.1002/jmri.22673.

[13] G. Thörmer, H.H. Bertram, N. Garnov, V. Peter, T. Schütz, E. Shang, M. Blüher, T. Kahn, H. Busse, Software for automated MRI-based quantification of abdominal fat and preliminary evaluation in morbidly obese patients, J. Magn. Reson. Imaging. 37 (2013) 1144-1150. https://doi.org/10.1002/jmri.23890.

[14] S. Estrada, R. Lu, S. Conjeti, X. Orozco-Ruiz, J. Panos-Willuhn, M.M.B. Breteler, M. Reuter, FatSegNet: A fully automated deep learning pipeline for adipose tissue segmentation on abdominal dixon MRI, Magn Reson Med. 83 (2020) 1471-1483. https://doi.org/10.1002/mrm.28022.

\section{AuTHORS}

\section{Sudha Devi B}

Research Scholar, , Dept. of Computer Science,

Nesamony Memorial Christian College, Marthandam,

Tamil Nadu, India.

\section{Dr.D.S Misbha}

Assistant Professor, Dept. of Computer Applications, Nesamony Memorial Christian College, Marthandam, Tamil Nadu, India.
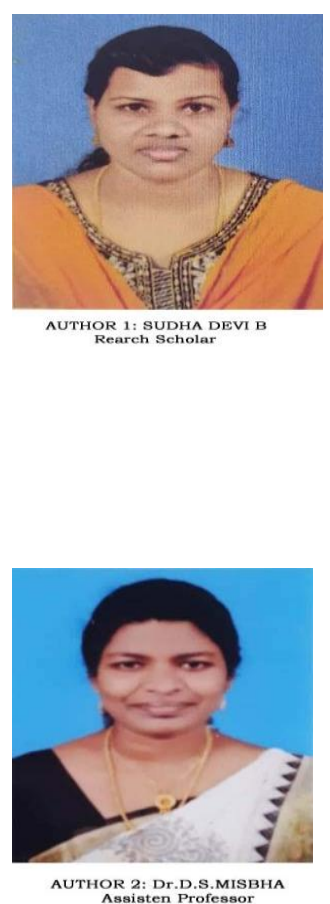